\title{
On the viability of achieving chiral separation through the optical manipulation of molecules
}

\author{
David L. Andrews* and David S. Bradshaw \\ School of Chemistry, University of East Anglia, Norwich Research Park, Norwich NR4 7TJ, U. K.
}

\begin{abstract}
Several different optical methods have recently been proposed for the potential separation of chiral molecules according to their intrinsic handedness. Applying fundamental symmetry and electrodynamical principles provides a perspective that casts doubt over the viability of some of the more extravagant claims. However there is a genuine basis for achieving chiral separation by using circularly polarized light to deliver chirally sensitive optical forces. The mechanism comes into play when molecules (or nanoscale particles) are optically trapped in a laser beam by forward Rayleigh scattering, as a result of trapping forces that depend on positioning within the beam profile. In such a setup, chiral molecules experience subtle additional forces associated with a combination of electric and magnetic transition dipoles; when circularly polarized light is used for the trapping, a discriminatory response can be identified that has the capacity to separate left- and righthanded molecular isomers. Here, clear differences can be observed between the behavior of isotropic liquids and poled solutions or liquid crystals. Detailed analysis provides an objective basis to assess new prospects for the recognition and differentiation of molecules with opposite chiral form, identifying and paving the way for future commercial applications.
\end{abstract}

Keywords: chirality in optical beams, optical activity, optical traps, circular polarization, helicity, optical forces, quantum electrodynamics

\section{INTRODUCTION}

Optical forces associated with chirality are presently receiving much attention. ${ }^{1-14}$ Research of this type commonly aims to achieve a discrimination of handedness, when a chiral molecule is irradiated with circularly polarized light. Identifying a different response for left- and right-handed input polarizations provides potential grounds for newly devised theoretical methods designed for the spatial separation of enantiomers (chiral molecules of opposite handedness) using a chiral force. This paper offers a more viable procedure, based on trapping, to achieve this kind of separation. Optical trapping and manipulation of molecules (and nanoscale particles) is a well-established technique involving the establishment of a trap due to the input of an off-resonant laser beam. ${ }^{15,16}$ In general, forward-Rayleigh scattering is the mechanism that describes such an event; the resultant optical force is dependent on the position of the molecule within the beam - as a result of the typically non-uniform intensity profile of the beam. Alternatively, the mechanism may be represented by the dynamic (ac) Stark effect, where an energy lowering of the molecular ground state arises through the alternating electric field of the input radiation. In quantum terms, both phenomena are identically described in terms of the annihilation and creation of identical photons, i.e. the instantaneous single absorption and single emission of photons with identical wavelengths within the transparent region of the molecule. Typically, the electric dipole-electric dipole (E1-E1) contribution is the leading contributor to such a mechanism but, in the trapping of chiral molecules, electric dipole-magnetic dipole (E1-M1) couplings need also to be considered; in fact, the latter, is the major factor in chiral discrimination studies. As such, in the two photon-molecule interactions of forward-Rayleigh scattering, the case of most interest is where one coupling is mediated by a transition electric dipole and the other by a transition magnetic dipole (Figure 1). Typically, the material response in a Rayleigh scattering mechanism is sufficiently described by an electronic polarizability involving transition electric dipoles, but the analogous E1-M1 polarizability tenor is required when considering chirality, as will be shown. The theory developed in this Proceedings is based on a quantum electrodynamical framework. 


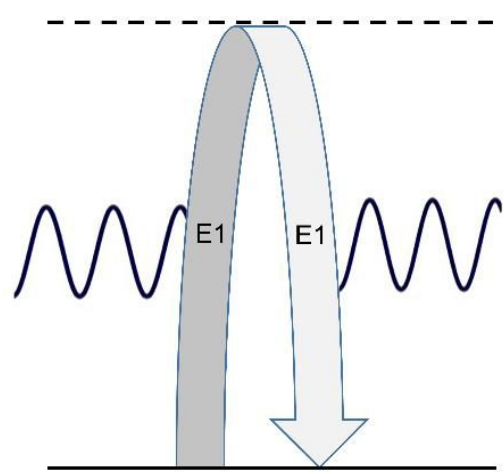

(a)

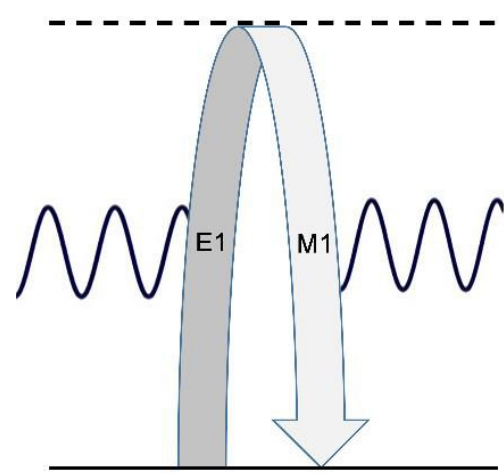

(b)

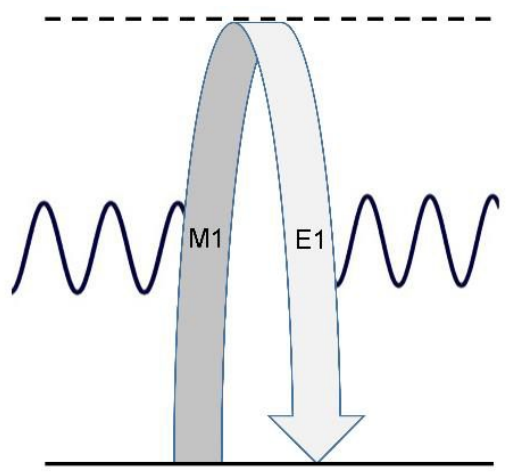

(c)

Figure 1. Schematic energy level diagrams depicting forward-Rayleigh scattering, relating to (a) conventional optical trapping, involving transition electric dipoles (E1) interactions, $(b)$ optical separation of chiral molecules, comprising an electric and magnetic dipole (M1) couplings and (c) vice-versa. Horizontal arrows denote concerted absorption and emission due to the input laser beam, associated with upward and downward electronic transitions from the molecular ground state (horizontal solid line) to a virtual state (dotted line).

\section{OPTICAL TRAPPING OF CHIRAL MOLECULES IN FIXED POSITIONS}

The physical observable of optical trapping is a potential energy (or energy shift), $\Delta E$, dependent on position $\mathbf{r}$, which may be defined in terms of identical initial and final states, denoted by $I$, and determined from second-order time-dependent perturbation theory, i.e.;

$$
\Delta E(\mathbf{r})=\operatorname{Re} M_{I I}(\mathbf{r}) \simeq \operatorname{Re}\left\{\sum_{R} \frac{\left\langle I\left|H_{\mathrm{int}}\right| R\right\rangle\left\langle R\left|H_{\mathrm{int}}\right| I\right\rangle}{E_{I}-E_{R}}\right\},
$$

where $R$ is an intermediate state, $E$ is the energy of the state denoted by the subscript and $H_{\text {int }}$ is the interaction Hamiltonian. Often the electric dipole approximation is applied but here, as stated in the introduction, consideration of the magnetic dipole moments is also required. In chirality studies, $H_{\text {int }}$ is thus given by;

$$
H_{\text {int }}=-\varepsilon_{0}^{-1} \boldsymbol{\mu} \cdot \mathbf{d}^{\perp}-\mathbf{m} \cdot \mathbf{b}
$$

where $\boldsymbol{\mu}$ and $\mathbf{m}$ are the electric and magnetic dipole moment operators, respectively, $\mathbf{d}^{\perp}$ and $\mathbf{b}$ are the transverse electric field and magnetic field operators, respectively; the well-known mode expansions of the latter are given elsewhere. ${ }^{17}$ The optical trapping force per chiral molecule is obtained from;

$$
\mathbf{F}(\mathbf{r})=-\operatorname{Re} \nabla M_{I I}(\mathbf{r})
$$

Assuming a circularly polarized input beam of either specific handedness, the potential energy of the optical trap is determined by insertion of the explicit form of equation (2) into the two relevant positions of equation (1) - representing the two light-matter interactions of Rayleigh scattering - so that the following expression is derived; ${ }^{18}$ 


$$
\Delta E^{(L / R)}(\mathbf{r})=\operatorname{Re}\left\{-\left(\frac{I(\mathbf{r})}{2 \varepsilon_{0} c^{2}}\right)\left(c \bar{e}_{i}^{(L / R)} \alpha_{i j} e_{j}^{(L / R)}+\bar{e}_{i}^{(L / R)} G_{i j} b_{j}^{(L / R)}+\bar{b}_{i}^{(L / R)} \bar{G}_{j i} e_{j}^{(L / R)}\right)\right\},
$$

where the first term corresponds to the E1-E1 interaction, and the other terms relate to the E1-M1 couplings. Moreover, $I(\mathbf{r})$ is the irradiance of the input beam, $\mathbf{e}^{(L / R)}$ and $\mathbf{b}^{(L / R)}$ are the circular polarization vectors of the electric and magnetic field, respectively - either left- or right-handed as denoted by the superscript - and $\alpha_{i j}$ is the polarizability tensor; $G_{i j}$ is an electric-magnetic tensor analogue ${ }^{19}$ to $\alpha_{i j}$ and its components are imaginary quantities. Determining an expression for optical discrimination, i.e. the difference in potential energy for left-handed compared to right-handed circular polarization, requires application of the identities $\mathbf{b}^{(L / R)}=\mp i \mathbf{e}^{(L / R)}$ and $\bar{e}_{i}^{(L)} e_{j}^{(L)}-\bar{e}_{i}^{(R)} e_{j}^{(R)}=i \varepsilon_{i j i m} \hat{k}_{k}$ so that equation (4) becomes;

$$
\Delta E^{(L)}(\mathbf{r})-\Delta E^{(R)}(\mathbf{r})=i\left(\frac{I(\mathbf{r})}{2 \varepsilon_{0} c^{2}}\right)\left(\bar{e}_{i}^{(L)} e_{j}^{(L)}+\bar{e}_{i}^{(R)} e_{j}^{(R)}\right)\left(G_{i j}(\omega)-\bar{G}_{j i}(\omega)\right)
$$

Here, following the symmetry of identical E1-E1 results for left- and right-handed polarizations, only the two E1-M1 terms (those involving the $G_{i j}$ tensor) remain. It has been assumed in equation (5) that discrimination arises when a single enantiomer is irradiated with circularly polarized light and its response to left- and right-handed polarizations differs. Although, significantly, identical outcomes occur for cases when a single circular polarization is applied to a left-handed chiral molecule relative to a right-handed enantiomer. The latter is important in connection with the optical response of a mixture of enantiomers, which is often produced in chemical synthesis. ${ }^{20}$ Equation (5), therefore, corresponds to a nonzero chiral trapping force for spatial separation of enantiomers in a fixed configuration.

\section{FULLY AND PARTIALLY TUMBLING SYSTEMS}

In this section, formulae are constructed for systems involving, for example, isotropic liquids and poled solutions (or liquid crystals) where individual molecules are allowed to freely or partially tumble in the laboratory frame. First an isotropic rotational-average is applied, in which each molecule is fully decoupled from the space-fixed frame into its own molecular frame; a detailed description on the method employed is found elsewhere. ${ }^{21,22}$ On applying an isotropic rotational average to equation (5), the following is found;

$$
\left\langle\Delta E^{(L)}(\mathbf{r})\right\rangle_{0}-\left\langle\Delta E^{(R)}(\mathbf{r})\right\rangle_{0}=-\left(\frac{2 I(\mathbf{r})}{3 \varepsilon_{0} c^{2}}\right) G_{\lambda \lambda}^{\prime}
$$

where $G_{\lambda \lambda}^{\prime}=\operatorname{Im}\left[G_{\lambda \lambda}\right]$, the Greek indices representing the molecular frame, and the subscript 0 denotes an isotropic rotational average. Considering a partial orientation of the molecules due to an applied static electric field, $\mathbf{E}_{0}$, requires the use of a weighting factor $-\boldsymbol{\mu}^{00} \cdot \mathbf{E}_{0}$, where $\boldsymbol{\mu}^{00}$ is a static electric dipole, within a Boltzmann factor $\exp \left(\boldsymbol{\mu}^{00} \cdot \mathbf{E}_{0} / k_{\mathrm{B}} T\right)$. A rotational average of this type ${ }^{23}$ is applied to equation (5), giving;

$$
\left\langle\Delta E^{(L)}\right\rangle-\left\langle\Delta E^{(R)}\right\rangle=-\left(\frac{I}{\varepsilon_{0} c^{2}}\right)\left(G_{\lambda \lambda}^{\prime}-\left\langle\hat{k}_{i} \hat{k}_{j} G_{i j}^{\prime} \mathrm{e}^{\frac{\mu^{00} \cdot \mathbf{E}_{0}}{k_{\mathrm{B}} T}}\right\rangle /\left\langle\mathrm{e}^{\frac{\mu^{00} \cdot \mathbf{E}_{0}}{k_{\mathrm{B}} T}}\right\rangle\right) .
$$


Here, the identity $\bar{e}_{i}^{(L)} e_{j}^{(L)}+\bar{e}_{i}^{(R)} e_{j}^{(R)} \equiv \delta_{i j}-\hat{k}_{i} \hat{k}_{j}$ is used. On completion of the Boltzmann-weighted average in equation (7), the following outcome for a partially tumbling system is derived;

$$
\left\langle\Delta E^{(L)}\right\rangle-\left\langle\Delta E^{(R)}\right\rangle=-\left(\frac{I}{\varepsilon_{0} c^{2}}\right)\left\{\frac{2}{3} G_{\lambda \lambda}^{\prime}+\left(1-\frac{3}{x} \operatorname{coth}(x)+\frac{3}{x^{2}}\right) P_{2}(\cos \theta)\left(G_{\lambda \mu}^{\prime} \hat{\mu}_{\lambda}^{00} \hat{\mu}_{\mu}^{00}-\frac{1}{3} G_{\lambda \lambda}^{\prime}\right)\right\}
$$

where $x=\boldsymbol{\mu}^{00} \cdot \mathbf{E}_{0} / k_{\mathrm{B}} T$ and $P_{2}(\cos \theta)$ is a Legendre polynomial, in which $\theta$ denotes the angle between $\hat{\mathbf{E}}_{0}$ and $\hat{\mathbf{k}}$.

\section{DISCUSSION}

This work reports a non-zero potential energy for chiral discrimination in an optical trap. The corresponding chiral force is derived from $\Delta \mathbf{F}(\mathbf{r}) \equiv \mathbf{F}^{(\mathrm{L})}(\mathbf{r})-\mathbf{F}^{(\mathrm{R})}(\mathbf{r})$, which is found from the potential energy via equation (3). Discriminatory results have been shown to arise for molecules in fixed orientations, isotropic liquids (where the molecules are free to tumble) and poled solutions (tumbling with respect to a static electric field). Of most interest are cases where light of a given circular polarization has a slightly different response when applied to a left-handed chiral molecule relative to a right-handed one - leading to the possibility of enantiomer separation. In the microscale, such optomechanical separation has been achieved experimentally, ${ }^{24}$ although difficulties arise on downscaling to molecular levels: for example, suggestions based on circular Bragg reflection ${ }^{25}$ prove problematic due to thermal effects.

Another potential setup, theoretically proposed by Cameron et al., ${ }^{10,11}$ involves the scattering of overlapped beams of orthogonal linear polarization by chiral molecules. However, from a fundamental photonic perspective, such a system cannot relate to a chiral force (or a discriminatory energy) since the initial and final states are not identical. ${ }^{26}$ This is readily demonstrated: since two beams are required for the proposed mechanism, and the process is elastic (no overall excitation or relaxation of molecular electronic state occurs) then any contributory mechanism has to involve a scattering event in which a photon is annihilated from one beam and a photon created into the other Since the initial and final radiation states of such a process are not identical, energy shifts cannot arise, and it becomes evident that there are no grounds for discriminatory forces to arise. Related chiral discriminatory systems have been proposed in the literature, although they lack the advantages of our optical trapping model. For example, the microwave spectroscopy method ${ }^{27}$ detects but does not separate the enantiomers, and applies to the gas phase only. Additionally, compared to a two-step switch, ${ }^{28,29}$ our suggestion requires no excitation of the molecules: resulting in a less energetic system with no time delays due to molecular recovery. The chiral discrimination effects we describe, therefore, appear promising as a model for enantiomer separation.

To determine a magnitude for such a chiral force, assuming the isotropic liquid case given by equation (6), we use the expression $\nabla F=-\left(2 G_{\lambda \lambda}^{\prime} / 3 \varepsilon_{0} c^{2}\right) \nabla I$. By using typical figures of $10^{9} \mathrm{~W} \mathrm{~cm}^{-2}$ and $800 \mathrm{~nm}$ for the beam intensity $I$ and width, respectively, and defining $G_{\lambda \lambda}^{\prime} / c=4 \pi \varepsilon_{0} d^{3} / 137$ assuming molecular dimensions $d$ in the $10 \mathrm{~nm}$ range, we discover that the chiral force is within the $10^{-15} \mathrm{~N}$ range. Comparing this to the thermodynamic force associated with molecular diffusion, against a concentration gradient (from Fick's first law), ${ }^{30}$ it transpires that a force of this scale should sustain a gradient of $\sim 0.5 \%$ in the differential concentrations of enantiomers with opposite handedness, $(\Delta C / C)$, per cycle. This represents a strong basis for achieving useful levels of separation on repeated cycling. The concentration figure might also be significantly increased, for example, by the use of laser wavelengths in the outer span of an optical absorption band, producing pre-resonance enhancement of the electric-magnetic polarizability tensor $G$.

\section{ACKNOWLEDGMENTS}

The authors would like to thank the Leverhulme Trust for funding this research. 


\section{REFERENCES}

[1] Zhao, R., Zhou, J., Koschny, T., Economou, E. N. and Soukoulis, C. M., "Repulsive Casimir force in chiral metamaterials," Phys. Rev. Lett. 103, 103602 (2009).

[2] Shang, Q. C., Wu, Z. S., Li, Z. J. and Li, H. A., "Radiation force on a chiral sphere by a Gaussian beam," Proc. SPIE 7845, 78452B (2010).

[3] Zhao, R., Koschny, T., Economou, E. N. and Soukoulis, C. M., "Chiral metamaterials reduce the attractive Casimir force," Proc. SPIE 7754, $77540 Z$ (2010).

[4] Zhao, R., Koschny, T., Economou, E. N. and Soukoulis, C. M., "Comparison of chiral metamaterial designs for repulsive Casimir force," Phys. Rev. B 81, 5 (2010).

[5] Butcher, D. T., Buhmann, S. Y. and Scheel, S., "Casimir-Polder forces between chiral objects," New J. Phys. 14, 113013 (2012).

[6] Hakami, J. and Zubairy, M. S., "Coherent control of Casimir force in a chiral medium," J. Phys. B: At. Mol. Opt. Phys. 45, 205502 (2012).

[7] Hu, Y. H., Mao, C. X. and Hu, H., "Chiral force between a simple model of chiral molecules and a copper block induced by an axion,” Chin. J. Phys. 50, 776-782 (2012).

[8] Canaguier-Durand, A., Hutchison, J. A., Genet, C. and Ebbesen, T. W., "Mechanical separation of chiral dipoles by chiral light," New J. Phys. 15, 123037 (2013).

[9] Shang, Q.-C., Wu, Z.-S., Qu, T., Li, Z.-J., Bai, L. and Gong, L., "Analysis of the radiation force and torque exerted on a chiral sphere by a Gaussian beam,” Opt. Express 21, 8677-8688 (2013).

[10] Cameron, R. P., Barnett, S. M. and Yao, A. M., "Discriminatory optical force for chiral molecules," New J. Phys. 16, 013020 (2014).

[11] Cameron, R. P., Yao, A. M. and Barnett, S. M., "Diffraction gratings for chiral molecules and their applications," J. Phys. Chem. A 118, 3472-3478 (2014).

[12] Canaguier-Durand, A. and Genet, C., "Chiral near fields generated from plasmonic optical lattices," Phys. Rev. A 90, 023842 (2014).

[13] Ding, K., Ng, J., Zhou, L. and Chan, C. T., "Realization of optical pulling forces using chirality," Phys. Rev. A 89, 063825 (2014).

[14] Wang, S. B. and Chan, C. T., "Lateral optical force on chiral particles near a surface," Nat. Commun. 5, 3307 (2014).

[15] Čižmár, T., Dávila Romero, L. C., Dholakia, K. and Andrews, D. L., "Multiple optical trapping and binding: New routes to self-assembly,” J. Phys. B: At. Mol. Opt. Phys. 43, 102001 (2010).

[16] Marago, O. M., Jones, P. H., Gucciardi, P. G., Volpe, G. and Ferrari, A. C., "Optical trapping and manipulation of nanostructures," Nat. Nanotechnol. 8, 807-819 (2013).

[17] Andrews, D. L. and Bradshaw, D. S., "Virtual photons, dipole fields and energy transfer: a quantum electrodynamical approach,” Eur. J. Phys. 25, 845-858 (2004).

[18] Bradshaw, D. S. and Andrews, D. L., "Chiral discrimination in optical trapping and manipulation," New J. Phys. 16, 103021 (2014).

[19] Craig, D. P. and Thirunamachandran, T., [Molecular Quantum Electrodynamics: An Introduction to RadiationMolecule Interactions], Dover Publications, Mineola, NY (1998). 
[20] Cossy, J. R., "The importance of chirality in drugs and agrochemicals," in [Comprehensive Chirality], H. Yamamoto and E. M. Carreira, eds. (Elsevier, Oxford, 2012), pp. 1-7.

[21] Ford, J. S. and Andrews, D. L., "One- and two-photon absorption in solution: The effects of a passive auxiliary beam,” J. Chem. Phys. 141, 034504 (2014).

[22] Friese, D. H., Beerepoot, M. T. P. and Ruud, K., "Rotational averaging of multiphoton absorption cross sections," J. Chem. Phys. 141, 204103 (2014).

[23] Andrews, D. L. and Harlow, M. J., "Phased and Boltzmann-weighted rotational averages," Phys. Rev. A 29, 27962806 (1984).

[24] Tkachenko, G. and Brasselet, E., "Optofluidic sorting of material chirality by chiral light," Nat. Commun. 5, 3577 (2014).

[25] Faryad, M. and Lakhtakia, A., "The circular Bragg phenomenon,” Adv. Opt. Photon. 6, 225-292 (2014).

[26] Bradshaw, D. S. and Andrews, D. L., "Interparticle interactions: Energy potentials, energy transfer, and nanoscale mechanical motion in response to optical radiation," J. Phys. Chem. A 117, 75-82 (2013).

[27] Patterson, D., Schnell, M. and Doyle, J. M., "Enantiomer-specific detection of chiral molecules via microwave spectroscopy," Nature 497, 475-477 (2013).

[28] Král, P., Thanopulos, I., Shapiro, M. and Cohen, D., "Two-step enantio-selective optical switch,” Phys. Rev. Lett. 90, 033001 (2003).

[29] Jia, W. Z. and Wei, L. F., "Distinguishing left- and right-handed molecules using two-step coherent pulses," J. Phys. B: At. Mol. Opt. Phys. 43, 185402 (2010).

[30] Atkins, P. W. and De Paula, J., [Atkins' Physical Chemistry], Oxford University Press, Oxford, 766 (2010).

*david.andrews@physics.org 